\title{
Differentiating Normal from Pathological Brain Ageing Using Standard Neuropsychological Tests
}

\author{
Sarah J. Wakefield ${ }^{1}$, William J. McGeown ${ }^{2}$, Michael F. Shanks ${ }^{1}$ and Annalena Venneri ${ }^{1,3, *}$ \\ ${ }^{I}$ Department of Neuroscience, University of Sheffield, UK, ${ }^{2}$ School of Psychological Sciences and Health, University of \\ Strathclyde, UK; ${ }^{3}$ IRCCS, Fondazione Ospedale San Camillo, Venice, Italy
}

\begin{abstract}
To diagnose Alzheimer's disease (AD) early, tests sensitive to neuropathology and insensitive to normal ageing are of greatest benefit. We used several neuropsychological tests to identify those best suited to distinguishing Mild Cognitive Impairment (MCI) and early AD from normal ageing. Impairments in long-term memory were found in older adults and these were even greater in MCI and $\mathrm{AD}$. Older adults outperformed young controls on category fluency and produced later acquired and less familiar words. Older adults also outperformed both patient groups on this task producing more words which were significantly later acquired, less familiar and less typical. Decline in long-term memory appears nonspecific and in the early stage of AD cannot help the differentiation between normal and pathological brain ageing. Normal ageing has no negative effects on verbal fluency, and impairment on this task signals not only established AD, but also its prodromal MCI stage.
\end{abstract}

Keywords: Age of acquisition, Alzheimer's disease, episodic memory, mild cognitive impairment, semantic memory, verbal fluency.

\section{INTRODUCTION}

A decline in some aspects of cognitive ability does occur with age, but sometimes signals a progressive neurodegenerative disease such as Alzheimer's Disease (AD). Memory impairments and declines of other cognitive skills are common in ageing, but do not necessarily imply progression to dementia due to AD [1]. When impairment is more marked, category of Mild Cognitive Impairment (MCI) has been variously defined [2]. MCI has a prevalence in community studies of $10-25 \%$ in the over 65 's [3] and about $10-15 \%$ incident cases of AD appear in MCI cohorts in the course of a year compared with $1-2 \%$ in the normal elderly population [2-4]. Several studies focusing on ancillary changes in neuroimaging and chemical biomarkers have been carried out to predict which patients with MCI will develop progressive cognitive decline [5]. These methods have not been fully validated and remain largely impracticable in clinical practice. There remains a need to improve clinical diagnostic methods to avoid missed or delayed diagnosis of $\mathrm{AD}$ and foster consistent management and treatment [6].New diagnostic definitions which should allow the identification of the disease more accurately in the prodromal and even preclinical stages with the help of available biomarkers have been developed $[7,8]$, and will also drive earlier clinical trial work and interventions. The existing literature is inconsistent because of variable research diagnostic criteria [9]. On this background, novel and reliable psychometric methods of predicting early disease presence and progression become increasingly relevant.

*Address correspondence to this author at the Department of Neuroscience, Medical School, Beech Hill Road, Sheffield, S10 2RX, UK;

Tel: +44 114 2713430; Fax: ????????????????;

E-mail: a.venneri@sheffield.ac.uk
Much previous research has focused on episodic memory ability and $\mathrm{AD}$, but significant impairment of episodic memory is present in some individuals who are ageing normally [10]. The diagnostic potential of changes in other cognitive abilities have therefore become of interest. Neuropsychological studies of patients with MCI, the prodromal stage of $\mathrm{AD}$, have shown additional impairments in verbal fluency, especially in category fluency $[11,12]$. The findings in prodromal AD reflect the evidence in established clinical AD where many studies have shown poorer performance in category than letter fluency tasks [13-15]. This weighting of impairment in category fluency seems to follow damage in areas that support semantic memory, in particular the perirhinal cortex and entorhinal cortex, structures which are vulnerable and damaged very early in the course of AD [16].

The assessment of semantic memory, therefore, provides one way of substantially increasing diagnostic confidence early in disease development. One reason for examining semantic memory is that, unlike the impairment of episodic memory in normal ageing, semantic memory seems less vulnerable to the effects of ageing [17]. Semantic memory decline is prevalent in patients with $\mathrm{AD}$ and that increased severity of disease results in a greater loss of semantic memory [18]. To test the integrity of semantic memory, fluency tasks are widely used and category fluency is especially suitable. This task involves the oral generation of as many responses as possible in specific categories (for example, 'Animals') within a 60 seconds limit. The ability of this test to discriminate normal from pathological ageing has led to the suggestion that it could be used as a one-minute mental status examination $[19,20]$. AD patients also perseverate more and include more intrusions and wrong category examples within their answers than healthy older adult controls [14]. 
Research has also shown that AD patients not only generate fewer exemplars than control participants on the category fluency task, but also generate fewer living than nonliving exemplars [21], Gender effects have also been shown on the fluency task. Moreno-Martinez et al [22] reported gender differences in elderly control participants and $\mathrm{AD}$ patients, but not in young control participants. These authors reported an effect of gender, with females producing more 'flowers' and 'vegetables' exemplars, while males produced more 'musical instruments'. Furthermore, among the AD patients, these authors noted a significant difference in both living ('insects' and 'trees') and non-living categories ('vehicles', 'tools' and 'musical instruments'), with the males producing more exemplars in both cases. This study also found age and disease effects. A reason put forward to explain these category specific differences was that the distinct anatomical regions supporting the production of the words were differentially damaged by the disease process [23].

To increase sensitivity and specificity of the category fluency task Forbes-McKay and colleagues [14] measured the lexical characteristics of the words produced in this test. These lexical attributes included Age of Acquisition (AoA), a measure of when the word was first learned. AD patients produced earlier acquired, more frequent and more typical words on the category fluency tasks than normal ageing controls [14]. Earlier acquired words are said to be more richly connected and embedded into cortical representation, while the cortical representation of later acquired words is more focused on temporal structures [24] and therefore more vulnerable to disruption caused by the spreading of AD pathology into temporal association cortex. Their more widespread cortical representations must facilitate the prompt retrieval of earlier acquired words, given their better connections with structures which are relatively preserved in $\mathrm{AD}$, and such words can be produced by AD patients even at a more advanced stage[25]. In a recent study, Biundo and colleagues [26] reported that AoA values were also able to identify MCI patients on the basis of the apolipoprotein $\varepsilon 4$ allele (ApoE $\left.\varepsilon_{4} 4\right)$ carrier status. Carriers produced significantly earlier acquired category exemplars.

The aim of the present study was to establish whether specific neuropsychological profiles could be identified using standard neuropsychological tests which would optimally differentiate normal from abnormal ageing in its early stage. A further aim was to test whether the age of acquisition effect reported by earlier studies of $\mathrm{AD}$ was present in normal ageing and if not, whether this would be a possible endophenotype of abnormal ageing.

\section{MATERIAL AND METHODS}

\subsection{Research Participants}

Retrospective data from patients attending an NHS Memory Clinic were included in the study. All patients gave consent for their clinical assessment scores to be included in an anonymised database for future research. They had psychiatric, neurological and extensive neuropsychological testing. The NINCDA-ADRDA clinical criteria were used to diagnose all patients with probable Alzheimer's Disease [27].Thirty probable AD patients aged between 52 and 91 years (mean age 69.00; SD 10.97) were selected for inclu- sion. They had an education level ranging from 9 to 20 years (mean education 11.83; SD 3.04), and their Mini Mental Status Examination (MMSE) [28] scores were between 12 and 27 (mean MMSE score 19.20; SD 4.05).

Thirty patients with a diagnosis of Mild Cognitive Impairment were also selected. Only patients whose mild deficits had a probable neurodegenerative aetiology were included. Other causes for their cognitive deficits were excluded after reviewing each patient's clinical history, MRI, and neuropsychological profile. Those who had a history indicative of vascular brain disease or had other vascular risk factors or had evidence of excessive vascular damage on the MRI (as determined by inspection of FLAIR sagittal and axial scans) were excluded. All patients diagnosed with MCI met the Peterson [29] criteria. The MCI patients were aged between 42 and 84 years (mean age 67.77; SD 10.53) and had levels of education ranging from 9 to 16 years (mean education 11.20; SD 1.90). The MCI patients' scores on the MMSE ranged between 24 and 30 (mean MMSE 26.57; SD 1.70).

Thirty healthy older adult controls were matched for age and education. A group of young controls were also recruited. The younger and older adult controls were selected from a large sample of participants who had been involved in a large standardisation study of a battery of neuropsychological tests and had undergone thorough background health screening before enrolment in that study. The older adult controls were aged between 48 years and 87 years (mean age 69.90; SD 10.24). Their education range was from 7 to 16 years of formal education (mean education 11.60; SD 2.06). Their MMSE scores were between 26 and 30 (mean MMSE score 28.70; SD 1.09). The young controls were aged between 18 years and 20 years (mean age 18.63; SD 0.67). Their education level ranged from 11 to 16 years of formal education (mean education 13.50; SD 1.28) and they achieved MMSE scores between 26 and 30 (mean MMSE score 28.70; SD 1.06). All controls had the same neuropsychological testing as the patients.

\subsection{Tasks and Procedure}

\subsubsection{Neuropsychological Battery}

All patients and controls had extensive neuropsychological assessment. The neuropsychological battery included the Mini Mental State Examination [28], tests of language comprehension [30] and naming by confrontation, tests of category and letter fluency, tests of short and long term memory (verbal and non-verbal) [31,32], tests of abstract reasoning $[31,33]$ and tests of attention. A full description of these tests can be found in [34]. Activities of daily living (ADL) and instrumental activities of daily living (IADL) were also assessed with formal scales [35], Of particular interest for the present study were the verbal fluency tasks.

\subsubsection{Category Fluency Task}

For the category fluency task, participants were asked to generate orally as many words from the categories of 'cities', 'animals' and 'fruits' as they could, in 60 second trials. Each category was performed in a separate trial. The number of correct words generated in each trial was recorded and perseverations (the same word said twice or more) were noted. 
Additional analyses were carried out for words' lexical semantic attributes.

\subsubsection{Word Attributes}

The following word attribute analyses included the categories of 'animals' and 'fruits' only (and did not include 'cities').

\subsubsection{Age of Acquisition (AoA)}

An AoA value was determined for each acceptable word produced in the category fluency task for the animal and fruit categories only. AoA values were obtained from ratings acquired by an earlier study (Biundo, 2010 University of Hulldoctoral dissertation, available at: https://hydra.hull.ac.uk/ resources/hull:5713). In that study, 150 healthy adult participants were recruited and split into age categories: 18-20 years; $21-30$ years; $31-40$ years; $41-50$ years; $51-60$ years; $61-70$ years; $>70$ years. Participants rated 366 animal examples and 110 fruit examples by estimating the age at which they learned each word. They were asked to state the age in whole years at which they learned each word. Word AoA values were obtained per age-group by calculating the harmonic mean of individual words in the two categories ('animals' and 'fruits'). These values were then used in the present study. Individual words were substituted with their corresponding AoA value taken from the database. An average AoA (from the words generated) could then be calculated for each person.

\subsubsection{Familiarity}

Word familiarity values were used to compute which group produced the most/least familiar exemplars of each category for the fruit and animal categories. Familiarity values had been acquired for each exemplar by Biundo (2010 University of Hulldoctoral dissertation). Participants rated, on a scale of 1 least familiar to 7 most familiar, how familiar they personally believed each particular animal and fruit was. Age-group harmonic mean values were calculated and the words produced by each participant were substituted with their corresponding familiarity value, and the mean calculated.

\subsubsection{Typicality}

Typicality refers to how representative a particular word is of a category. Typicality values had also been acquired by Biundo (2010, University of Hulldoctoral dissertation). The participants rated, on a scale of 1 least typical to 7 most typi$\mathrm{cal}$, how typical each particular animal and fruit was to that category. Age group harmonic mean values were calculated for each word and these typicality values were then substituted for the words generated, and individual mean values calculated.

\subsubsection{Letter Fluency Task}

The letter fluency task involved participants orally generating words beginning with specific letters - in this case the letters $\mathrm{P}, \mathrm{L}$ and $\mathrm{F}$. The letters were performed in separate trials that lasted 60 seconds each. The task was scored by the amount of correct words generated in each trial. Proper nouns were not counted. Perseverations were noted. Additional analyses were carried out for type of words produced.

\section{RESULTS}

\subsection{Demographics}

There were no age differences between the older adult controls and either patient group, or between the patient groups. The young controls differed significantly from the older adult controls at $(\mathrm{p}<0.011)$ in years of formal education. No other significant differences in education were found between groups.

\subsection{Neuropsychological Tests}

Analyses ofindividual test scores from the neuropsychological test battery completed by patients and controls were done using one-way ANOV As. A Bonferroni corrected significance level was used and $\mathrm{p}$ value significance threshold was set at $p<0.0031$ ). Table 1 shows the mean scores and the standard deviations achieved by each group on the neuropsychological tests used in the battery. Significant between groups differences are highlighted by symbols.

\subsection{Normal Ageing Effects}

Normal ageing effects were found on tasks assessing new learning and visuospatial long-term memory, with the young controls outperforming the older adult controls. A significant difference between the younger and older adult groups was found on the Delay component of Rey's Complex Figure and on the Verbal Paired Associates $(\mathrm{p}<0.0001)$. No significant difference was seen on the Copy component of Rey's Complex Figure $(\mathrm{p}=0.673)$.On the Confrontation Naming task, there was a reversed ageing effect with the older adult controls outperforming the young controls $(p<0.001)$ and naming correctly more pictures.

\subsection{Pathological Ageing Effects}

The older adult controls significantly outperformed the AD patient group on most tasks within the battery. The biggest differences were on tasks assessing global cognition (MMSE: $\mathrm{p}<0.0001$ ), attention (Stroop: error interference $\mathrm{p}<0.0001$ and time interference $\mathrm{p}<0.0001)$, language comprehension (Token task: $\mathrm{p}<0.0001$ ), and semantic association (Pyramid and Palm Trees: $\mathrm{p}<0.001$ ), although differences on other tasks were also seen (see Table 1). The older adult controls also had significantly higher scores than the MCI patients on some tasks within the battery including the delay component of Rey's Complex Figure (Delay $p<0.0001$ ).

In the patient groups, the MCI patients outperformed the $\mathrm{AD}$ patients on the Digit Cancellation task: $\mathrm{p}<0.0001$ ) (see Table 1 for details).

\subsection{Verbal Fluency tasks}

\subsubsection{Category Fluency}

\subsubsection{Number of Words}

A significant difference was found on the category fluency task between the groups in number of words produced on all three category trials: $[\mathrm{F}(3,116)=46.484$, $\mathrm{p}<0.0001]_{\text {, There }}$ was no significant difference between the control groups in the overall number of words produced. There were, however, significant differences between the 
Table 1. Neuropsychological performance.

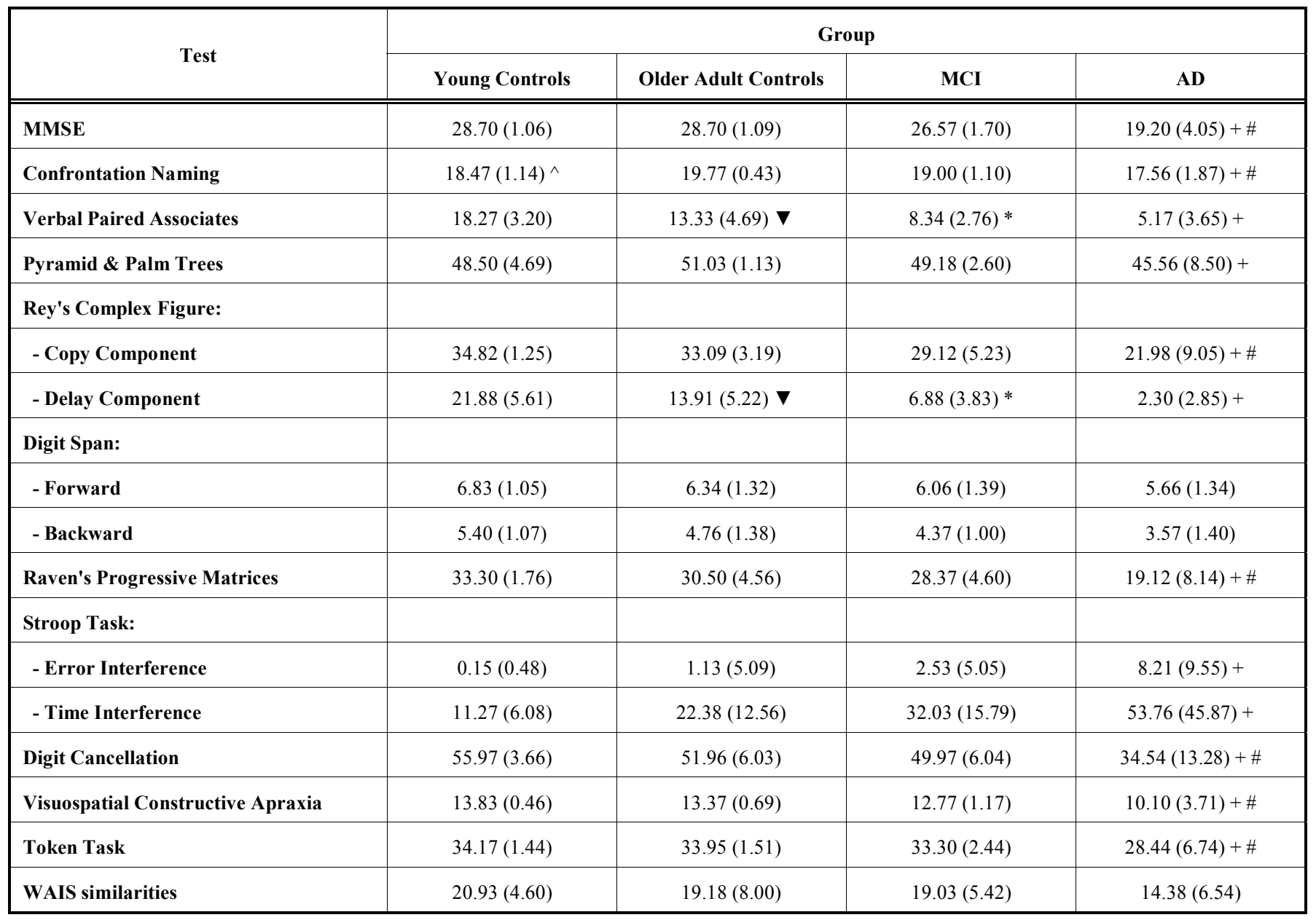

Mean performance (SD) and significant differences among young and older adult control groups, MCI and AD patients on tests within the neuropsychological battery. Key:

$\nabla$ significant difference, young controls $>$ older adult controls

$\wedge$ significant difference, older adult controls $>$ young controls

* significant difference, older adult controls $>$ MCI patients

+ significantdifference, older adult controls $>$ AD patients

\# significant difference, MCI patients $>$ AD patients

older adult controls and both patient groups as the former outperformed both MCI and AD patients at $\mathrm{p}<0.0001$ level (see Table 2). The MCI patients produced significantly more words than the AD group, $\mathrm{p}<0.004$ (see Table 2).

\subsubsection{Analysis of Lexical Characteristics of Words}

Items produced in the 'animals and fruits' categories were analysed. Bonferroni corrected significance level was used and $p$ value threshold was set a $p<0.017$. There were significant differences amongst groups for AoA $[\mathrm{F}(3,116)=$ $11.081, \mathrm{p}<0.0001]$, familiarity $[\mathrm{F}(3,116)=5.748$, $\mathrm{p}=0.001$ land Typicality $[\mathrm{F}(3,116)=9.641, \mathrm{p}<0.0001$ [values.

The older adult controls produced words that were higher in AoA value (i.e., acquired later in life) at $\mathrm{p}<0.0001$ (see Table 2) and less familiar words at $p=0.003$ than the young controls. No differences between the control groups were found on typicality $(\mathrm{p}=0.071)$.

The older adult controls produced significantly higher AoA words than the MCI and AD patient groups $(\mathrm{p}<0.002$ and $\mathrm{p}<0.003$, respectively) (see Table 2 ). Older adult con- trols produced words that were less familiar than those of the $\mathrm{AD}$ group at $\mathrm{p}<0.017$, but not less familiar than those produced by the MCI group $(p=0.158)$ (see Table 2). Similarly, older adult controls produced words which were less typical than those of the AD patients only $(\mathrm{p}<0.005)$ ( Table 2). There were no significant differences between the lexical characteristics of the words produced by the patient groups.

\subsection{Letter Fluency}

\subsubsection{Number of Words}

A significant difference was found on the letter fluency task amongst the groups on total number of words $[\mathrm{F}(3,115)$ $=12.066, \mathrm{p}<0.0001]_{\text {. }}$ The number of words produced by young and older adult controls did not differ. There was a significant difference between the scores of the older adult controls and of both patient groups, who produced significantly fewer words $(p<0.05$ for MCI and $p<0.0001$ for AD)(see Table 3). The MCI patients performed better than the AD patients $(\mathrm{p}<0.05)$. 
Table 2. Semantic fluency and lexical performance.

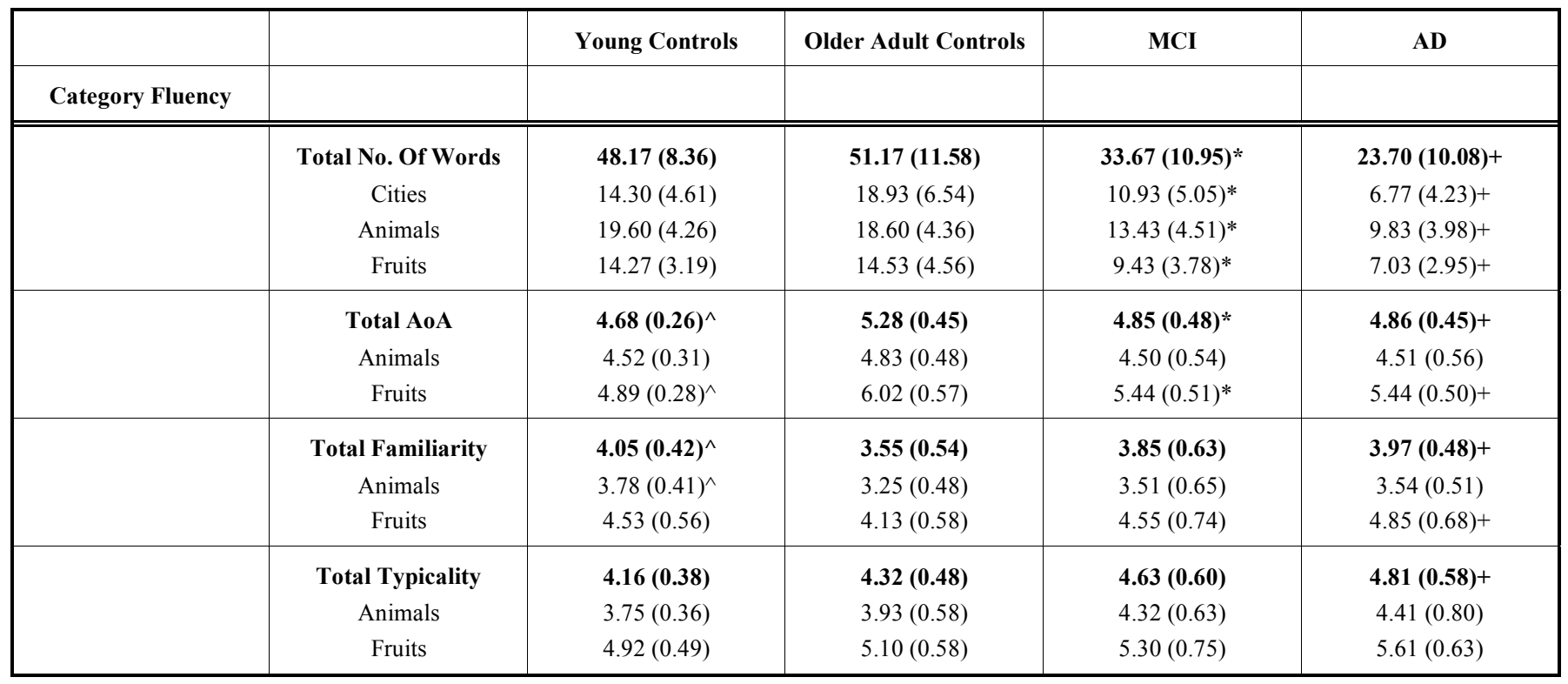

Mean performance of young and older adult control groups, MCI and AD patients on the semantic fluency task and lexical analysis. Key:

$\wedge$ significant difference, older adult controls $>$ young controls

* significant difference, older adult controls $>$ MCI patients

+ significant difference, older adult controls $>\mathrm{AD}$ patients

\# significant difference, $\mathrm{MCI}$ patients $>\mathrm{AD}$ patients

Table 3. Phonemic fluency and type of word performance

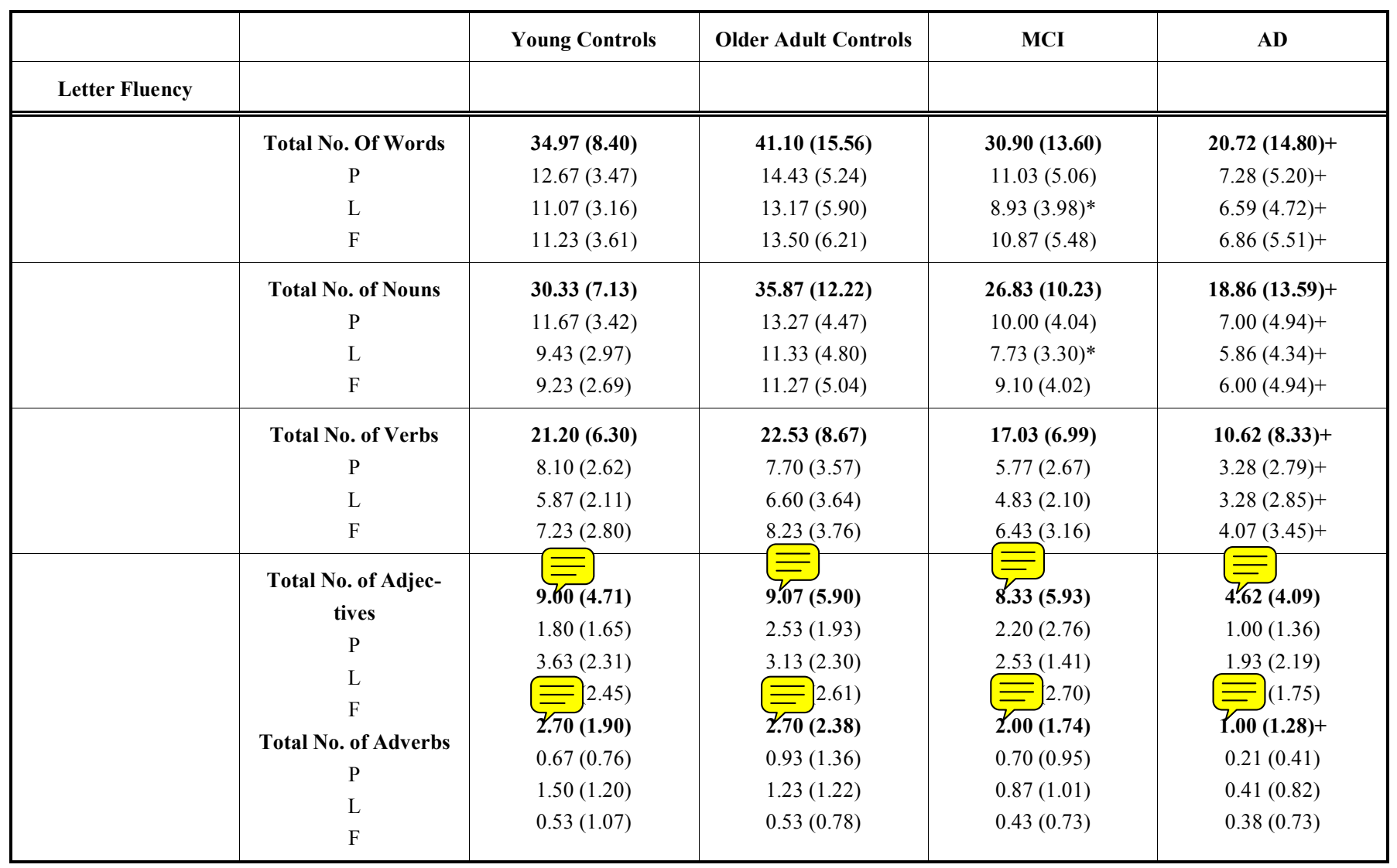

Mean performance of young and older adult control groups, MCI and AD patients on the phonemic fluency task and lexical analysis. Key:

* significant difference, older adult controls $>$ MCI patients

+ significant difference, older adult controls $>$ AD patients

\# significant difference, MCI patients $>$ AD patients 


\subsubsection{Analyses of Type of Word}

Analyses of type of word, i.e., noun, verb, adjective and adverb, showed significant differences amongst groups on number of nouns and verbs produced (Bonferroni correction was applied and significance level adjusted at $\mathrm{p}<0.012)$ : Nouns - $[\mathrm{F}(3,115)=12.267, \mathrm{p}<0.0001] ;$ Verbs $[\mathrm{F}(3,115)=$ 14.483, $\mathrm{p}<0.0001]$; Adjectives $[\mathrm{F}(3,115)=4.688, \mathrm{p}<0.005]$; and Adverbs $[\mathrm{F}(3,115)=5.425, \mathrm{p}<0.002]$. There were no significant differences between young and older adult controls however. The older adult controls produced more nouns than the AD patients $(p<0.0001$ and they also produced more verbs $(\mathrm{p}<0.0001)$ than the AD patients (See Table 3 ).

\section{DISCUSSION}

In this study the older adult and young controls performed at similar levels in most tasks included in the neuropsychological battery, and in some tasks older adults outperformed the young controls (e.g., Confrontation Naming). Normal ageing effects were seen on tasks which assess aspects of long term memory such as the Verbal Paired Associates and the delay component of Rey's Complex Figure. It follows that low scores on episodic long-term memory tasks do not necessarily indicate that an individual is ageing abnormally. Detailed analysis of the category fluency data showed no age related decline, and therefore poor performance on this task should be taken as a potential disease indicator, prompt further assessment and potentially trigger longitudinal follow up. Other tasks also showed sensitivity to early abnormal cognitive decline (e.g., Rey's Complex Figure), and were also able to identify differences in severity between MCI and AD (e.g., Stroop Task). The additional item analyses of both fluency tasks did, as predicted, differentiate normal from abnormal decline in both $\mathrm{AD}$ and $\mathrm{MCI}$ patients and might aid the difficult distinction between these states of abnormal ageing [36]. This study suggests that score profiles derived from a broad neuropsychological test battery can contribute most to the distinction between healthy cognitive ageing and MCI, healthy cognitive ageing and $\mathrm{AD}$, and $\mathrm{MCI}$ and $\mathrm{AD}$. This approach, if applied routinely, would increase the accuracy of early stage clinical diagnosis.

The tasks within the neuropsychological battery which assess semantic memory and knowledge can also differentiate across the groups. The Pyramid and Palm Trees test distinguished the older adult controls from the $\mathrm{AD}$ patients, while the category fluency task separated older adult controls from both $\mathrm{MCI}$ and $\mathrm{AD}$ patients, and the milder impairment (MCI patients) from the more severe group(AD patients). The older adult controls' performance on the Pyramid and Palm Trees test was even better than the young controls, showing this aspect of semantic memory remains unaffected by ageing. In contrast, episodic memory declines significantly in normal ageing.

In a study of possible confounding demographic factors, Nyberg et al [37] reported that age explained variance in performance of normal individuals (age range 35-80) on tests of episodic memory, but not on tests of semantic memory. Episodic memory, therefore, shows a decline with increasing age, and this explains why younger participants achieved better scores on episodic memory tests than the older partici- pants and no age related decline was found for semantic memory. Episodic and semantic memory tests also show better performance in the domain of remote recall than with recent memories [38]. These aspects of memory may be differentially affected by ageing because semantic tasks rely to a greater extent on remote memory, while episodic memory tasks involve new learning and the temporal gradients observed in retrograde memory appears to parallel an age of acquisition effect in retrograde memory [39].

Category fluency has already been shown to differentiate normal from pathological ageing [14]. That study examined whether controls could produce later acquired words simply because they named more words in the 60 second trials. The differential power of the category fluency task persisted when only the first five words from each category were analysed. AoA, frequency and typicality still allowed separation of the groups, but these word parameters did not stage disease severity. Forbes-McKay and colleagues [14] work shows this effect clearly, and indicates that there is no linear effect of AoA, familiarity or typicality with increasing severity of the disease. Instead, this appears to be a specific disease effect that characterises the individual from early in the disease process, even in prodromal $\mathrm{AD}$, and continues throughout. One interpretation might be that the AoA effect reflects very early impairment of brain structures supporting semantic retrieval or representation $[16,40]$. The AoA effect in category fluency is associated with atrophy in regions of the medial temporal lobes, including the perirhinal cortex, in the mild stage of $\mathrm{AD}$ [41] and $\mathrm{MCI}$ [12], as are other aspects of semantic retrieval in these groups [42].

Research has also suggested that some frontal processes have an impact on category fluency tasks [43, 44]. The role of executive control, however, is probably over estimated, and neuroimaging evidence shows that the association with frontal structures is limited and that proficiency on this task is more reliant on temporal cortex and cerebellum [41, 45]. In letter fluency however, a task that requires more executive control, reliance on frontal structures is more important and again there is neuroimaging evidence to substantiate this claim $[41,46]$. Patient data also support this view. Mild patients with AD are less impaired on letter fluency than category fluency [47], while these tasks are equally affected in the early stage of front temporal dementia [48].

Noage related decline was found on the letter fluency task; young and older adult controls performed similarly on all measures of this task. The older adult controls produced more words than the $\mathrm{AD}$ patients, although their scores did not differ from the MCI patients. The older adult controls also produced more nouns and verbs than the $\mathrm{AD}$ group, but not more than the MCI. There were no differences between patient groups. Item analysis of words in the letter fluency task showed that $\mathrm{AD}$ patients produced fewer verbs than older adult controls. The ability to produce verbs is more impaired in pathological ageing of the Alzheimer-type [49], and patients would be expected to differ most on the production of this type of word than other type, such as nouns, adjectives or adverbs. Many researchers have suggested that verbs are not only harder to process than nouns, but they are also learned later in life than nouns [49]. This would suggest a sort of AoA effect on the letter fluency, similar to that seen 
on the category fluency task. Just like with the categories of 'animals' and 'fruits', whereby the normal ageing individuals (i.e., older adult control group) produced words that were, on average, later acquired in life than those produced by the pathological ageing groups of MCI patients and $\mathrm{AD}$ patients, this process can also be seen in the words produced by the different groups in the letter fluency task. The pathological ageing groups (MCI and $\mathrm{AD}$ ), on this task, produced fewer verbs than the older adult controls, indicating that these are less intact in the patient's memory than nouns, just as the later acquired category fluency examples are less intact and so less likely to be produced by the pathological ageing patients.

This study demonstrates the wealth of information that can be extracted from standard neuropsychological tests and show the more specific features of word production can be used to interpret performance, aiding identification of normal and abnormal cognitive decline. There was dissociation between age and disease effects in episodic and semantic memory measures in the same samples of patients and controls. On further investigation into the conversion rate in the MCI group, $33 \%$ of the whole group had converted since the time from their first assessment, which is in line with the reported rate in other publications. This study has implications for the assessment of patients presenting at memory clinics for specialist investigation of memory complaints.

\section{CONFLICT OF INTEREST}

The authors confirm that this article content has no conflict of interest.

\section{ACKNOWLEDGEMENTS}

Declared none.

\section{REFERENCES}

[1] Petersen, R.C., Mild Cognitive Impairment. New England Journal of Medicine, 2011.364(23): p. 2227-2234.

[2] Petersen, R.C., Mild cognitive impairment as a diagnostic entity. Journal of Internal Medicine, 2004. 256(3): p. 183-194.

[3] Plassman, B.L., et al., Prevalence of cognitive impairment without dementia in the United States. Annals of Internal Medicine, 2008. 148(6): p. 427-434.

[4] Farias, S.T., et al., Progression of Mild Cognitive Impairment to Dementia in Clinic- vs Community-Based Cohorts. Archives of neurology, 2009. 66(9): p. 1151-1157.

[5] Petersen, R.C. and C.R. Jack, Imaging and Biomarkers in Early Alzheimer's Disease and Mild Cognitive Impairment. Clinical Pharmacology \& Therapeutics, 2009. 86(4): p. 438-441.

[6] Seltzer, B., Cholinesterase inhibitors in the clinical management of Alzheimer's disease: importance of early and persistent treatment. The Journal of international medical research, 2006. 34(4): p. 33947.

[7] Dubois, B., et al., Research criteria for the diagnosis of Alzheimer"s disease: revising the NINCDS-ADRDA criteria. Lancet neurology, 2007. 6(8): p. 734-746.

[8] McKhann, G.M., et al., The diagnosis of dementia due to Alzheimer's disease: Recommendations from the National Institute on Aging-Alzheimer's Association workgroups on diagnostic guidelines for Alzheimer's disease. Alzheimers \& Dementia, 2011. 7(3): p. 263-269.

[9] Stephan, B.C.M., et al., Diagnosing Mild Cognitive Impairment (MCI) in clinical trials: a systematic review. BMJ open, 2013. 3(2).

[10] Ciaramelli, E., R. Lauro-Grotto, and A. Treves, Dissociating episodic from semantic access mode by mutual information measures: Evidence from aging and Alzheimer's disease. Journal of Physiology-Paris, 2006. 100(1-3): p. 142-153.

[11] Hodges, J.R., S. Erzinclioglu, and K. Patterson, Evolution of cognitive deficits and conversion to dementia in patients with mild cognitive impairment: A very-long-term follow-up study. Dementia and geriatric cognitive disorders, 2006. 21(5-6): p. 380-391.

[12] Venneri, A., et al., The Neuroanatomical Substrate of LexicalSemantic Decline in MCI APOE epsilon 4 Carriers and Noncarriers. Alzheimer Disease \& Associated Disorders, 2011. 25(3): p. 230-241.

[13] Capitani, E., et al., Mirror asymmetry of Category and Letter fluency in traumatic brain injury and Alzheimer's patients. Neuropsychologia, 2009. 47(2): p. 423-429.

[14] Forbes-McKay, K.E., et al., The age of acquisition of words produced in a semantic fluency task can reliably differentiate normal from pathological age related cognitive decline. Neuropsychologia, 2005. 43(11): p. 1625-1632.

[15] Pasquier, F., et al., Verbal fluency in dementia of frontal lobe type and dementia of Alzheimer type. Journal of neurology, neurosurgery, and psychiatry, 1995. 58(1): p. 81-4.

[16] Braak, H. and E. Braak, Neuropathological Staging of AlzheimerRelated Changes. Acta neuropathologica, 1991. 82(4): p. 239-259.

[17] Craik, F.I.M., Memory Changes in Normal Aging. Current Directions in Psychological Science, 1994. 3(5): p. 155-158.

[18] Westmacott, R., et al., The contribution of autobiographical significance to semantic memory: evidence from Alzheimer's disease, semantic dementia, and amnesia. Neuropsychologia, 2004. 42(1): p. 25-48.

[19] Cummings, J.L., The one-minute mental status examination. Neurology, 2004. 62(4): p. 534-5.

[20] Duff-Canning, S.J., et al., Diagnostic utility of abbreviated fluency measures in Alzheimer disease and vascular dementia. Neurology, 2004. 62(4): p. 556-62.

[21] Zannino, G.D., et al., Category-specific naming deficit in Alzheimer's disease: the effect of a display by domain interaction. Neuropsychologia, 2007. 45(8): p. 1832-9.

[22] Moreno-Martinez, F.J., K.R. Laws, and J. Schulz, The impact of dementia, age and sex on category fluency: greater deficits in women with Alzheimer's disease. Cortex, 2008. 44(9): p. 1256-64.

[23] Brambati, S.M., et al., The anatomy of category-specific object naming in neurodegenerative diseases. J Cogn Neurosci, 2006. 18(10): p. $1644-53$.

[24] Ellis, A.W., et al., Traces of vocabulary acquisition in the brain: Evidence from covert object naming. NeuroImage, 2006. 33(3): p. 958-968.

[25] Steyvers, M. and J.B. Tenenbaum, The large-scale structure of semantic networks: statistical analyses and a model of semantic growth. Cogn Sci, 2005. 29(1): p. 41-78.

[26] Biundo, R., et al., Influence of APOE Status on Lexical-Semantic Skills in Mild Cognitive Impairment. Journal of the International Neuropsychological Society, 2011. 17(3): p. 423-430.

[27] Mckhann, G., et al., Clinical-Diagnosis of Alzheimers-Disease Report of the Nincds-Adrda Work Group under the Auspices of Department-of-Health-and-Human-Services Task-Force on Alzheimers-Disease. Neurology, 1984. 34(7): p. 939-944.

[28] Folstein, M.F., S.E. Folstein, and P.R. McHugh, "Mini-mental state". A practical method for grading the cognitive state of patients for the clinician. J Psychiatr Res, 1975. 12(3): p. 189-98.

[29] Petersen, R.C., et al., Practice parameter: Early detection of dementia: Mild cognitive impairment (an evidence-based review) Report of the Quality Standards Subcommittee of the American Academy of Neurology. Neurology, 2001. 56(9): p. 1133-1142.

[30] De Renzi, E. and P. Faglioni, Normative Data and Screening Power of a Shortened Version of Token Test. Cortex; a journal devoted to the study of the nervous system and behavior, 1978. 14(1): p. 4149.

[31] Wechsler, D., Wechsler Adult Intelligence Scale-III. 1997, San Antonio: The Psychological Corporation.

[32] Rey, A., L'examen clinique en psychologie. 1964, Paris: Presses Universitaires de France.

[33] Raven, J.C., Coloured Progressive Matrices Sets A, Ab, B. Manual Sections $1 \&$ 2. 1995, Oxford: Oxford Psychologists Press.

[34] Lezak, M.D., Howieson, D.-B., Bigler, E.-D., Tranel, D., Neuropsychological Assessment. 5th Edition ed. 2012: Oxford University Press. 
[35] Lawton, M.P. and E.M. Brody, Assessment of Older People - SelfMaintaining and Instrumental Activities of Daily Living. Gerontologist, 1969. 9(3p1): p. 179-\&.

[36] Petersen, R.C., Morris, C.-M., Clinical Features, in Mild Cognitive Impairment: Aging to Alzheimer's Disease, R.C. Petersen, Editor. 2003, Oxford Press: New York. p. 15-39.

[37] Nyberg, L., et al., Age differences in episodic memory, semantic memory, and priming: Relationships to demographic, intellectual, and biological factors. Journals of Gerontology Series BPsychological Sciences and Social Sciences, 1996. 51(4): p. P234P240.

[38] Small, J.A. and N. Sandhu, Episodic and semantic memory influences on picture naming in Alzheimer's disease. Brain and language, 2008. 104(1): p. 1-9.

[39] Ivaniou, A., Cooper, J.M., Shanks, M.F., and Venneri, A.Patterns of impairment in autobiographical memory in the degenerative dementias constrain models of memory ${ }_{2}$ Neuropsychologia, 2006. 44: p.1936-1955.

[40] Braak, H. and E. Braak, Diagnostic criteria for neuropathologic assessment of Alzheimer's disease. Neurobiology of aging, 1997a. 18(4): p. S85-S88.

[41] Venneri, A., et al., The anatomical bases of semantic retrieval deficits in early Alzheimer's disease. Neuropsychologia, 2008. 46(2): p. 497-510.
[42] Hirni, D.I., et al., Distinct neuroanatomical bases of episodic and semantic memory performance in Alzheimer's disease. Neuropsychologia, 2013. 51(5): p. 930-937.

[43] Gardini, S., et al., Brain Structural Substrates of Semantic Memory Decline in Mild Cognitive Impairment. Current Alzheimer research, 2013. 10(4): p. 373-389.

[44] Szmalec, A., A. Vandierendonck, and E. Kemps, Response selection involves executive control: evidence from the selective interference paradigm. Mem Cognit, 2005. 33(3): p. 531-41.

[45] Leggio, M.G., et al., Phonological grouping is specifically affected in cerebellar patients: a verbal fluency study. Journal of Neurology Neurosurgery and Psychiatry, 2000. 69(1): p. 102-106.

[46] Costafreda, S.G., et al., A systematic review and quantitative appraisal of fMRI studies of verbal fluency: Role of the left inferior frontal gyrus. Human brain mapping, 2006. 27(10): p. 799-810.

[47] Pestell, S., et al., Quality of spelling breakdown in Alzheimer's disease is independent of disease progression. Journal of sclinical and experimental neuropsychology, 2000. 22(5): p. 599-612.

[48] Rascovsky, K., et al., Disparate letter and semantic category fluency deficits in autopsy-confirmed frontotemporal dementia and Alzheimer's disease. Neuropsychology, 2007. 21(1): p. 20-30.

[49] Matzig, S., et al., Noun and verb differences in picture naming: past studies and new evidence. Cortex, 2009. 45(6): p. 738-58. 